\title{
Resonant Fermi gases with a large effective range
}

\author{
A. Schwenk, 1 and C.J. Pethick ${ }^{2, \text { U }}$ \\ ${ }^{1}$ Nuclear Theory Center, Indiana University, Bloomington, IN 47408 \\ ${ }^{2}$ NORDITA, Blegdamsvej 17, DK-2100 Copenhagen Ø, Denmark
}

\begin{abstract}
We calculate the equation of state of a Fermi gas with resonant interactions when the effective range is appreciable. Using an effective field theory for large scattering length and large effective range, we show how calculations in this regime become tractable. Our results are model-independent, and as an application, we study the neutron matter equation of state at low densities of astrophysical interest $0.002 \mathrm{fm}^{-3}<\rho<0.02 \mathrm{fm}^{-3}$, for which the interparticle separation is comparable to the effective range. We compare our simple results with those of conventional many-body calculations.
\end{abstract}

The properties of dilute Fermi gases when all two-body scattering lengths $a_{\mathrm{s}}$ are large compared to the interparticle separation $r_{\mathrm{s}}$ and the range of the interaction $R$ is small compared with $r_{\mathrm{s}}$ are universal, in the sense that properties do not depend on details of the interparticle interaction [1]. This is because the only dimensionful scale is the Fermi momentum $k_{\mathrm{F}}$, and the corresponding energy scale is the Fermi energy $\varepsilon_{\mathrm{F}}=k_{\mathrm{F}}^{2} /(2 m), m$ being the fermion mass. Consequently all macroscopic observables are given by appropriate powers of $k_{\mathrm{F}}$ or $\varepsilon_{\mathrm{F}}$ multiplied by universal factors. For instance, for two spin states with equal populations the energy per particle $E / N$ of cold gases of, e.g., ${ }^{6} \mathrm{Li},{ }^{40} \mathrm{~K}$ atoms or neutrons under these conditions is given by

$$
\frac{E}{N}=\xi\left(\frac{E}{N}\right)_{\text {free }}=\xi \frac{3 k_{\mathrm{F}}^{2}}{10 m}
$$

where the universal factor $\xi$ is a number.

Resonant, dilute Fermi gases were realized for the first time by O'Hara et al. in 2002 [2]. The factor $\xi$ has been determined experimentally by a number of methods [2, 3, [4, 5] in atomic gases where the interaction may be tuned by controlling the magnetic field. The extraction of the equation of state leads to $\xi=0.51 \pm 0.04[3], \xi \approx 0.7[4]$ and $\xi=0.27_{-0.09}^{+0.12}[5]$, for temperatures in units of the Fermi temperature $T / T_{\mathrm{F}} \approx 0.05$ (except for $T / T_{\mathrm{F}} \approx 0.6$ in [4]). To date, the most reliable theoretical results are from $T=0$, fixed-node Green's function Monte Carlo simulations, $\xi=0.44 \pm 0.01$ [6] and $\xi=0.42 \pm 0.01$ [7].

The purpose of this letter is to consider the neutron gas as an example of a Fermi system with a large scattering length. For neutrons, the scattering length is unnaturally large and the currently accepted value is $a_{\mathrm{nn}}=-18.5 \pm 0.3 \mathrm{fm}$ (for a recent review on the experimental situation see [8]). This is to be compared with the range of nuclear interactions, which is given by the mass of the lightest exchange particle, the pion, and thus $R \sim 1 / m_{\pi} \approx 1.4 \mathrm{fm}$. The effective range $r_{\mathrm{e}}$ is expected to be approximately charge independent, and therefore we take the neutron-neutron effective range to be $r_{\mathrm{nn}}=2.7 \mathrm{fm}$, the same as the neutron-proton one $[9]$. Consequently, the neutron effective range is also significant, with $r_{\mathrm{e}} m_{\pi} \approx 2$.
For neutrons, the validity of the universal equation of state applies to densities with $k_{\mathrm{F}} r_{\mathrm{e}} \ll 1$. This restricts neutron densities to $\rho=k_{\mathrm{F}}^{3} /\left(3 \pi^{2}\right)<10^{-4} \mathrm{fm}^{-3}$, which is just below neutron drip density $\rho_{\text {nd }}=2.3 \cdot 10^{-4} \mathrm{fm}^{-3} \sim$ $10^{-3} \rho_{0}$ at which neutrons become unbound. Here $\rho_{0}=$ $0.16 \mathrm{fm}^{-3}$ is the saturation density of symmetric nuclear matter. Therefore, it is important to generalize the equation of state to resonant Fermi gases with an appreciable effective range. In the regime $k_{\mathrm{F}} r_{\mathrm{e}} \sim 1$, the energy per particle can be expressed as in Eq. (1) but with a systemspecific factor $\xi\left(k_{\mathrm{F}} r_{\mathrm{e}}\right)$,

$$
\frac{E}{N}=\xi\left(k_{\mathrm{F}} r_{\mathrm{e}}\right) \frac{3 k_{\mathrm{F}}^{2}}{10 m} .
$$

This is the case for neutron matter at subnuclear densities $\rho<\rho_{0} / 10$. For the extension Eq. (2) to be valid, the contributions to the energy from higher partial waves and many-body forces must be negligible. This ensures that neutron densities with $k_{\mathrm{F}} r_{\mathrm{e}} \sim 1$ do not probe further details of nuclear forces. In addition, the effective range expansion truncated at $r_{\mathrm{e}}, k \cot \delta(k)=-1 / a_{\mathrm{s}}+r_{\mathrm{e}} k^{2} / 2$, must describe the phase shifts $\delta(k)$ for S-wave scattering up to relative momenta $k \sim k_{\mathrm{F}}$. The extension of the universal energy to $k_{\mathrm{F}} r_{\mathrm{e}} \sim 1$ is also valid for trapped Fermi gases where both the scattering length and the effective range are tuned to large values. In this letter, we calculate $\xi\left(k_{\mathrm{F}} r_{\mathrm{e}}\right)$ for resonant Fermi gases with $k_{\mathrm{F}} r_{\mathrm{e}} \gtrsim 1$ and explain how this regime becomes theoretically tractable.

For neutron matter, the non-S-wave contributions to the energy per particle can be estimated from the Hartree-Fock results shown in Fig. 1 The Hartree-Fock calculations are based on the model-independent twonucleon interaction $V_{\text {low } k}$ for different values of the momentum cutoff $\Lambda$. The evolution of $V_{\text {low } k}$ with the cutoff follows a renormalization group (RG) equation that guarantees that nucleon-nucleon phase shifts are cutoff independent. We have previously found that the evolution of all large-cutoff nuclear interactions to $\Lambda \lesssim 2.1 \mathrm{fm}^{-1}$ leads to the same low-momentum interaction $V_{\text {low } k}[10]$. Varying the cutoff gives an approximate measure of higherorder contributions beyond Hartree-Fock and the effects of omitted many-body forces, since both are needed to obtain cutoff-independent results. We find in Fig. \that the low-density equation of state is cutoff-independent 


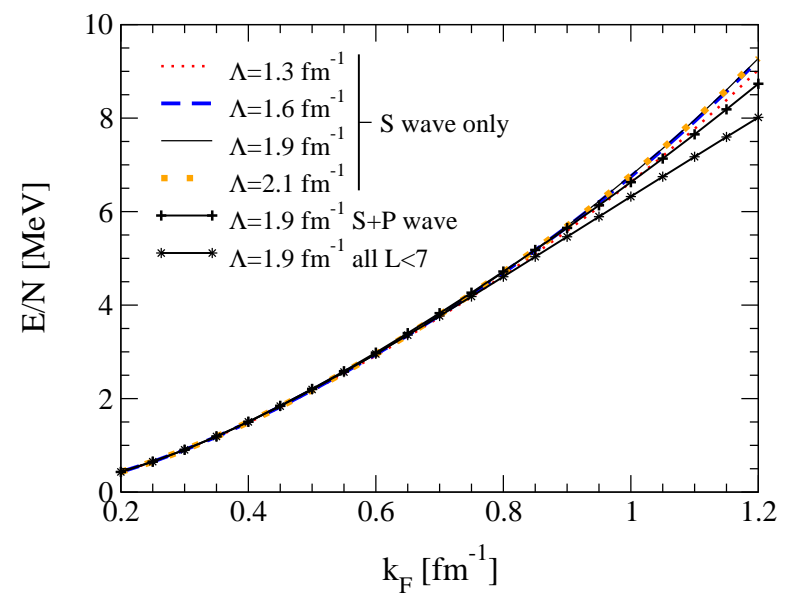

FIG. 1: Hartree-Fock results for the energy per particle of neutron matter calculated from $V_{\text {low } k}$ for different cutoffs.

for $k_{\mathrm{F}} \lesssim 1.0 \mathrm{fm}^{-1}$, with negligible $l \geqslant 1$ contributions for $k_{\mathrm{F}} \lesssim 0.8 \mathrm{fm}^{-1}$. In addition, the effective range expansion describes the ${ }^{1} \mathrm{~S}_{0}$ phase shifts well up to relative momenta $k<1.0 \mathrm{fm}^{-1}$, with a deviation of $-0.4,0.2$ and 2.1 degrees from the empirical phase shifts $57.7,48.6$ and 39.5 degrees for $k=0.4,0.6$ and $0.8 \mathrm{fm}^{-1}$ respectively. Consequently, the equation of state of neutron matter is given by Eq. (2) for $k_{\mathrm{F}} r_{\mathrm{e}} \lesssim 2$.

We calculate the equation of state Eq. (2) using an effective field theory (EFT) for large scattering length and large effective range. EFT offers a systematic ap- proach to interactions at low energies, and therefore our results are model-independent. Under the conditions of interest, both the scattering length and effective range are low-momentum scales $1 / a_{\mathrm{s}} \sim 1 / r_{\mathrm{e}} \sim Q$. The corresponding EFT is realized by introducing a di-fermion field $d$ [11] (for a review see Ref. [12]) with the lowestorder Lagrangian density given by (in units $\hbar=m=1$ )

$$
\begin{aligned}
\mathcal{L} & =\psi^{\dagger}\left(i \partial_{0}+\frac{\nabla^{2}}{2}\right) \psi-d^{\dagger}\left(i \partial_{0}+\frac{\nabla^{2}}{4}-\Delta\right) d \\
& -g\left(d^{\dagger} \psi \psi+d \psi^{\dagger} \psi^{\dagger}\right) .
\end{aligned}
$$

Here $\psi$ denotes the fermion field, and $\Delta$ and $g$ are lowenergy constants which describe the propagation of the di-fermion field and its coupling to two fermions respectively. We also note that $i \partial_{0}+\nabla^{2} / 4$ is the operator for the two-body energy corrected for the center-of-mass motion. The EFT Lagrangian Eq. (31) was introduced for nucleon-nucleon scattering in Ref. 11] following work due to Weinberg [13]. In the di-fermion EFT, the $T$ matrix for two-body scattering depends only on the energy $E$ of the interacting particles in the center-of-mass system,

$$
T(E)=\frac{g^{2}}{\Delta-E-g^{2} I_{0}(E+i \epsilon)},
$$

with $I_{0}(E)=\int_{0}^{\Lambda} d^{3} p /(2 \pi)^{3}\left(E-p^{2}\right)^{-1}$. This corresponds to summing diagrams where the di-fermion propagator (represented as a double line) is dressed by fermion loops,
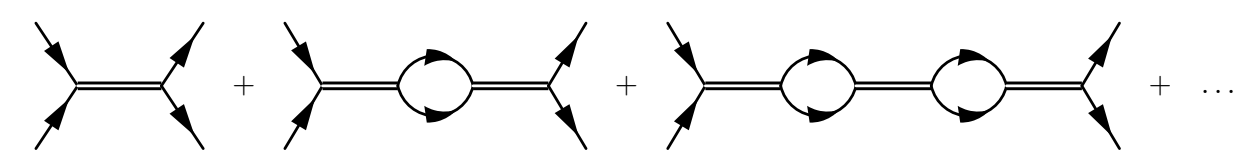

$I_{0}(E)$ denotes the loop integral regulated by a momentum cutoff $\Lambda$ and the on-shell $T$ matrix is given by $4 \pi T^{-1}\left(E=k^{2}\right)=1 / a_{\mathrm{s}}-r_{\mathrm{e}} k^{2} / 2+i k$, which can equivalently be obtained from an energy-dependent potential,

$$
V(E)=\frac{g^{2}}{\Delta-E},
$$

which follows after integrating out the di-fermion field. Matching the lowest-order low-energy constants $\Delta$ and $g$ to the effective range expansion yields

$$
\frac{\Delta}{g^{2}}=\frac{1}{4 \pi a_{\mathrm{s}}}-\frac{\Lambda}{2 \pi^{2}} \quad \text { and } \quad \frac{1}{g^{2}}=\frac{r_{\mathrm{e}}}{8 \pi}-\frac{1}{2 \pi^{2} \Lambda} .
$$

Consequently, for systems with large scattering length and large effective range the potential $V(E)$ scales at low energies $E \sim Q^{2}$ as $V(E) \sim\left(Q+\Lambda+Q^{2} / \Lambda\right)^{-1}$. The cutoff generates higher-order terms $\sim k^{4} / \Lambda^{3}$ in $T^{-1}\left(E=k^{2}\right)$, which are suppressed for $k / \Lambda \ll 1$. Therefore, we consider the lowest-order EFT with large cutoffs. This is not required for $V_{\text {low } k}$, since the RG generates all higherorder contact interactions necessary to maintain cutoffindependent two-body observables. If one rescales the di-fermion field by $d \rightarrow g d$, the interaction terms become coupling-independent and the kinetic term acquires a factor $1 / g^{2}$. For large cutoffs $1 / g^{2} \sim r_{\mathrm{e}}$, and therefore the di-fermion field is an auxiliary field for positive effective range, even if there is a two-body bound state with $a_{\mathrm{s}}>0$. Integrating out the $d$ field gives the standard contact interaction EFT. For negative effective range the di-fermion kinetic term has the normal sign and the Lagrangian Eq. (3) is the atom-molecule model. The great advantage of the present formulation is that it enables one to study systems with either positive or negative $r_{\mathrm{e}}$.

Next, we study the scaling of diagrammatic contributions to the energy. Particle-particle loops are restricted 
only by the cutoff, and therefore these scale as $\sim \Lambda$ for large cutoffs. By contrast, particle-hole and holehole intermediate states scale with the low-momentum scale $\sim Q$. Particle-hole loops, for instance, scale with the density of states $\sim k_{\mathrm{F}}$. Since the lowest-order interaction scales as $V(E) \sim\left(Q+\Lambda+Q^{2} / \Lambda\right)^{-1}$, cutoff inde- pendence for large cutoffs minimally requires summing particle-particle ladders with Pauli blocking, such that the $\Lambda^{-1}$ scaling of the potential cancels with each loop $\sim \Lambda$ [14. Neglecting terms which vanish for large cutoffs, we find for $T_{\text {med }}$, the on-shell in-medium $T$ matrix

$$
T_{\text {med }}\left(E=k^{2} ; P\right)=4 \pi\left[\frac{1}{a_{\mathrm{s}}}-\frac{r_{\mathrm{e}} k^{2}}{2}-\frac{k_{\mathrm{F}}+\frac{P}{2}}{\pi}+\frac{k}{\pi} \log \left(\frac{k_{\mathrm{F}}+\frac{P}{2}+k}{k_{\mathrm{F}}+\frac{P}{2}-k}\right)+\frac{k^{2}+\frac{P^{2}}{4}-k_{\mathrm{F}}^{2}}{\pi P} \log \left(\frac{\left(k_{\mathrm{F}}+\frac{P}{2}\right)^{2}-k^{2}}{k_{\mathrm{F}}^{2}-\frac{P^{2}}{4}-k^{2}}\right)\right]^{-1},
$$

where $\mathrm{P}$ denotes the particle-pair momentum and we have neglected the imaginary part, as the latter vanishes for $k<\sqrt{k_{\mathrm{F}}^{2}-P^{2} / 4}$ and therefore does not contribute to the energy. The resulting in-medium $T$ matrix can be viewed as an effective interaction and scales as $T_{\text {med }}\left(E=k^{2} ; P\right) \sim Q^{-1}$ independent of the cutoff. In addition to the scattering length and effective range terms, the inverse effective interaction acquires a densitydependent contribution $-\left(k_{\mathrm{F}}+P / 2\right) / \pi$ and two logarithmic terms. The first logarithmic term includes the BCS singularity for $P=0$ and $k=k_{\mathrm{F}}$. The resulting contribution to the energy is integrable and generally small. For neutron matter, realistic calculations of the superfluid pairing gap including polarization effects give $\Delta / \varepsilon_{\mathrm{F}} \sim 0.1$ for densities with $k_{\mathrm{F}} r_{\mathrm{e}} \sim 1$ [15]. We therefore expect corrections to the energy per particle $\xi_{\mathrm{sf}}\left(k_{\mathrm{F}} r_{\mathrm{e}}\right) \approx-5 / 8\left(\Delta / \varepsilon_{\mathrm{F}}\right)^{2} \approx-0.01$ due to pairing in this regime. The second logarithmic term in Eq. (8) is regular, and for $P=0$ it simplifies to $-k_{\mathrm{F}} / \pi$.

For large effective range, interactions are weaker at higher momenta and the effect on the in-medium $T$ matrix can be understood qualitatively by inserting average values for the momenta $k^{2}=3 / 10 k_{\mathrm{F}}^{2}$ and $P^{2}=6 / 5 k_{\mathrm{F}}^{2}$ into Eq. (8). With this qualitative estimate, the average effective interaction for resonant Fermi gases is given by

$$
\overline{T_{\mathrm{med}}}=-\frac{4 \pi}{U_{0} k_{\mathrm{F}}+\frac{3 r_{\mathrm{e}} k_{\mathrm{F}}^{2}}{20}}=-\frac{4 \pi}{U_{0} k_{\mathrm{F}}} \frac{1}{1+0.27 k_{\mathrm{F}} r_{\mathrm{e}}},
$$

with $U_{0}=0.56$. In the universal regime with $\frac{k_{\mathrm{F}} r_{\mathrm{e}}}{T_{\mathrm{m}}} \ll$ 1 , the average effective interaction is given by $\overline{T_{\text {med }}}=$ $-4 \pi /\left(U_{0} k_{\mathrm{F}}\right)$. The remaining contributions to the energy are of particle-hole or hole-hole nature, and scale as $Q \sim$ $k_{\mathrm{F}}$. In this EFT it is thus necessary to sum all diagrams, as the effects of each additional vertex $\sim k_{\mathrm{F}}^{-1}$ combined with the additional loop $\sim k_{\mathrm{F}}$ are of order one.

For Fermi gases with large effective range, the loop scaling is unchanged but additional $T_{\text {med }}$ vertices are now suppressed by $\left(1+0.27 k_{\mathrm{F}} r_{\mathrm{e}}\right)^{-1}$. This implies that corrections to the Hartree-Fock equation of state with $T_{\text {med }}$ as effective interaction come with powers of $\left(1+0.27 k_{\mathrm{F}} r_{\mathrm{e}}\right)^{-1}$ relative to the universal case. In perturbation theory,

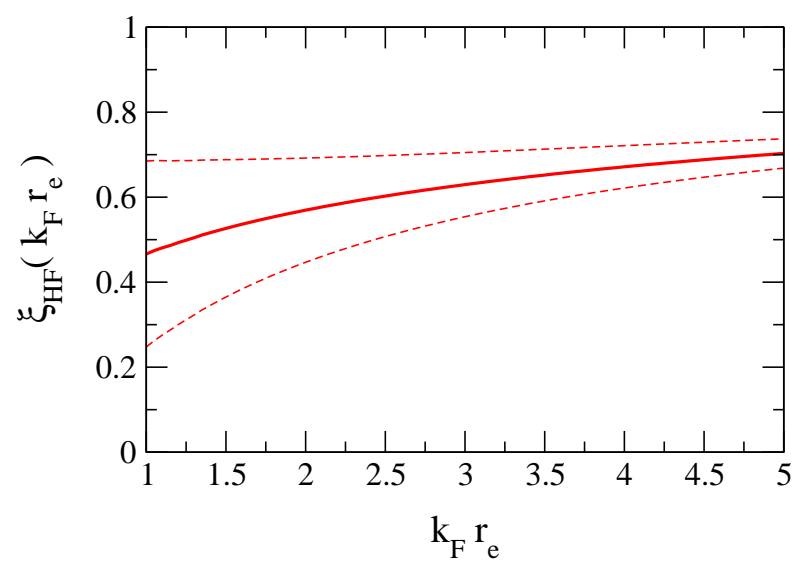

FIG. 2: Result for $\xi_{\mathrm{HF}}\left(k_{\mathrm{F}} r_{\mathrm{e}}\right)$ (solid line) which describes the equation of state of resonant Fermi gases with large effective range $k_{\mathrm{F}} r_{\mathrm{e}} \gtrsim 1$. The dashed lines provide an error estimate for $\xi_{\mathrm{HF}}$ as discussed in the text.

particle-particle-irreducible contributions to the energy enter in third order. With $\overline{T_{\text {med }}}=4 \pi \overline{a_{\text {med }}}$, we estimate the leading non-ladder corrections to the $\xi\left(k_{\mathrm{F}} r_{\mathrm{e}}\right)$ obtained from $T_{\text {med }}$ at the Hartree-Fock level as $\Delta \xi=$ $0.077\left(U_{0}\left(1+0.27 k_{\mathrm{F}} r_{\mathrm{e}}\right)\right)^{-3}$ [16]. This only provides a qualitative estimate, since we evaluated the in-medium $T$ matrix for average particle configurations.

To a good approximation, the equation of state of resonant Fermi gases with $k_{\mathrm{F}} r_{\mathrm{e}} \gtrsim 1$ can therefore be calculated from $T_{\text {med }}$ at the Hartree-Fock level,

$$
\begin{aligned}
\xi_{\mathrm{HF}}\left(k_{\mathrm{F}} r_{\mathrm{e}}\right) & =1+\frac{5}{2 \pi^{2} k_{\mathrm{F}}^{5}} \int_{0}^{2 k_{\mathrm{F}}} P^{2} d P \int_{0}^{\sqrt{k_{\mathrm{F}}^{2}-\frac{P^{2}}{4}}} k^{2} d k \\
& \times T_{\text {med }}\left(k^{2} ; P\right) \min \left[1, \frac{k_{\mathrm{F}}^{2}-k^{2}-\frac{P^{2}}{4}}{k P}\right],
\end{aligned}
$$

with $1 / a_{\mathrm{s}}=0$ in Eq. (8). Based on the arguments given above, we expect corrections to $\xi$ beyond Hartree-Fock of order $\Delta \xi=0.22-0.03$ and $\xi_{\mathrm{sf}} \approx-0.01$ due to pairing for densities with $k_{\mathrm{F}} r_{\mathrm{e}}=1-5$. Our results for $\xi_{\mathrm{HF}}\left(k_{\mathrm{F}} r_{\mathrm{e}}\right)$ are shown in Fig. 2 with the theoretical error estimate. It is intriguing that we find $\xi_{\mathrm{HF}}(1) \approx 0.47$, which is close 


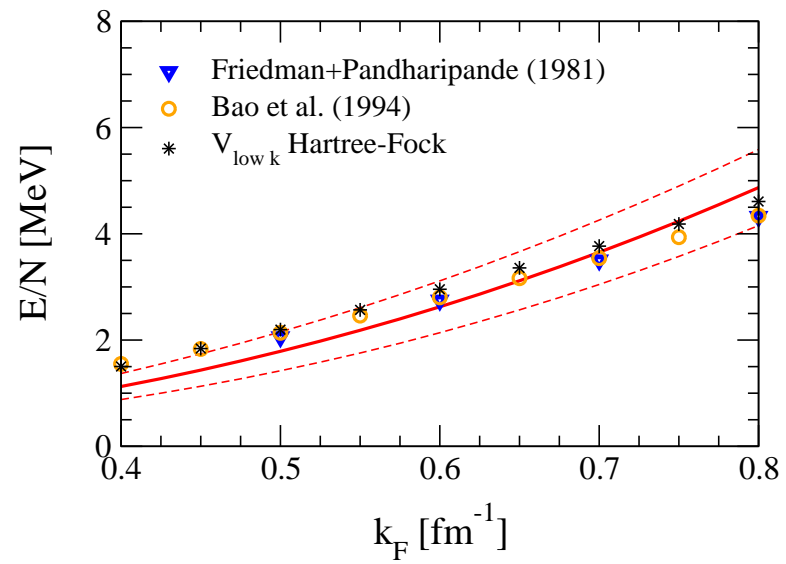

FIG. 3: Comparison of the energy per particle for neutron matter calculated from Eq. (10) for $a_{\mathrm{s}}=-18.5 \mathrm{fm}$ and $r_{\mathrm{e}}=$ $2.7 \mathrm{fm}$ (solid line) to Fermi hypernetted-chain (triangles) [17] and Brueckner results (circles) [18]. We also show the $V_{\text {low }}$ Hartree-Fock results of Fig. 1] The dashed lines provide an error estimate for $\xi_{\mathrm{HF}}$.

to the universal factor $\xi=0.44 \pm 0.01[6]$. This implies a cancellation of higher-order contributions for $k_{\mathrm{F}} r_{\mathrm{e}} \ll 1$.

For $k_{\mathrm{F}} r_{\mathrm{e}} \gg 1$, the effective interaction is of the or$\operatorname{der} \overline{T_{\text {med }}} \sim-\left(r_{\mathrm{e}} k_{\mathrm{F}}^{2}\right)^{-1}$ and interaction effects disappear for infinite effective range. In the latter regime, $\xi \approx 1+C /\left(k_{\mathrm{F}} r_{\mathrm{e}}\right)$, with a coefficient $C<0$ of order one. Consequently, $\xi \rightarrow 1$ only for very large effective ranges. We find $\xi_{\mathrm{HF}}(10)=0.79$ and $\xi_{\mathrm{HF}}(100)=0.96$. If the effective range is negative, the average interaction in the medium is greater than for $r_{\mathrm{e}}=0$, and higher-order terms are only reduced for very large $\left|k_{\mathrm{F}} r_{\mathrm{e}}\right| \gg 1$.

Finally, we take into account a large, but finite scattering length, and present in Fig. 3 results for the equation of state of neutron matter in the regime $k_{\mathrm{F}} r_{\mathrm{e}} \sim 1$. This is straightforward by including the $1 / a_{\mathrm{s}}$ term in Eq. (8). Our results are model-independent and constrain neutron matter at subnuclear densities $0.002 \mathrm{fm}^{-3}<\rho<$ $0.02 \mathrm{fm}^{-3}$, which is of relevance to the physics of neutron stars and supernovae. We also compare our neutron matter results to many-body calculations using Fermi hypernetted-chain techniques 17 or Brueckner theory [18]. As can be seen from Fig. 3 all microscopic results lie within our theoretical error estimates.

In summary, we have calculated the equation of state of resonant Fermi gases with large effective range $k_{\mathrm{F}} r_{\mathrm{e}} \gtrsim 1$ in the di-fermion EFT, with particular attention to neutron matter at subnuclear densities. In the lowest-order EFT, cutoff independence minimally requires the resummation of particle-particle ladders [13], which leads to an effective interaction that becomes weak for all $k_{\mathrm{F}} r_{\mathrm{e}} \gtrsim 1$. The $k_{\mathrm{F}} r_{\mathrm{e}}$ dependence was used to show how resonant Fermi gases with large effective range, such as low-density neutron matter, are theoretically tractable and to estimate the error of our results. Neutron matter equations of state obtained using conventional many-body approaches are consistent with our model-independent di- fermion EFT results within these errors. For $k_{\mathrm{F}} r_{\mathrm{e}} \sim 1$, the energy is found to be close to the universal regime.

We thank Georg Bruun, Dick Furnstahl, Chuck Horowitz and Daniel Phillips for useful discussions. The work of AS is supported by the US DOE under Grant No. DE-FG02-87ER40365 and the NSF under Grant No. PHY-0244822.

* E-mail: schwenk@indiana.edu

$\dagger$ E-mail: pethick@nordita.dk

[1] G.F. Bertsch posed the properties of Fermi gases in this limit as a many-body-theory challenge problem at MBX (2001). The first studies were reported by G.A. Baker, Int. J. Mod. Phys. B15, 1314 (2001); H. Heiselberg, Phys. Rev. A63, 043606 (2001).

[2] K.M. O'Hara, S.L. Hemmer, M.E. Gehm, S.R. Granade and J.E. Thomas, Science 298, 2179 (2002).

[3] M.E. Gehm, S.L. Hemmer, S.R. Granade, K.M. O'Hara and J.E. Thomas, Phys. Rev. A68, 011401(R) (2003); J. Kinast, A. Turlapov, J.E. Thomas, Q. Chen, J. Stajic and K. Levin, Science 307, 1296 (2005).

[4] T. Bourdel, J. Cubizolles, L. Khaykovich, K.M.F. Magalhaes, S. Kokkelmans, G.V. Shlyapnikov and C. Salomon, Phys. Rev. Lett. 91, 020402 (2003).

[5] M. Bartenstein, A. Altmeyer, S. Riedl, S. Jochim, C. Chin, J.H. Denschlag and R. Grimm, Phys. Rev. Lett. 92, 120401 (2004) and cond-mat/0412712

[6] J. Carlson, S.-Y. Chang, V.R. Pandharipande and K.E. Schmidt, Phys. Rev. Lett. 91, 050401 (2003); S.Y. Chang, J. Carlson, V.R. Pandharipande and K.E. Schmidt, Phys. Rev. A70, 043602 (2004).

[7] G.E. Astrakharchik, J. Boronat, J. Casulleras and S. Giorgini, Phys. Rev. Lett. 93, 200404 (2004).

[8] A. Gardestig and D.R. Phillips, nucl-th/0501049

[9] The neutron-proton effective range parameters are wellconstrained from the Nijmegen 1993 partial-wave analysis (PWA93): $a_{\mathrm{np}}=-23.768 \pm 0.006 \mathrm{fm}$ and $r_{\mathrm{np}}=$ $2.68 \pm 0.01 \mathrm{fm}$ (M.C.M. Rentmeester, private communication).

[10] S.K. Bogner, T.T.S. Kuo and A. Schwenk, Phys. Rept. 386, 1 (2003).

[11] D.B. Kaplan, Nucl. Phys. B494, 471 (1997); S.R. Beane and M.J. Savage, Nucl. Phys. A694, 511 (2001).

[12] D.R. Phillips, Czech. J. Phys. 52, B49 (2002).

[13] S. Weinberg, Phys. Rev. 130, 776 (1963).

[14] This resummation is not necessary if one evolves the large-cutoff theory to smaller cutoffs using the RG, see S.K. Bogner, A. Schwenk, R.J. Furnstahl and A. Nogga, Nucl. Phys. A in press, nucl-th/0504043

[15] A. Schwenk, B. Friman and G.E. Brown, Nucl. Phys. A713, 191 (2003); see also Fig. 2 in A. Schwenk, nucl-th/0411070

[16] The third-order non-ladder contributions to the energy have been evaluated by Baker, see Eq. (4.84) in G.A. Baker, Rev. Mod. Phys. 43, 479 (1971). Note that for $r_{\mathrm{e}}=0, \overline{T_{\mathrm{med}}}$ leads to $\xi_{\mathrm{HF}}=0.36$ and $\Delta \xi=0.45$.

[17] B. Friedman and V.R. Pandharipande, Nucl. Phys. A361, 502 (1981).

[18] G. Bao, L. Engvik, M. Hjorth-Jensen, E. Osnes and E. Østgaard, Nucl. Phys. A575, 707 (1994). 